\title{
Chlamydia trachomatis infection in dizygotic twins delivered by caesarean section
}

\author{
THOMAS A BELL
}

From the Departments of Pediatrics and Epidemiology, University of Washington, Seattle, Washington, USA

SUMMARY Chlamydia trachomatis was isolated from the conjunctiva, pharynx, and rectum of one 10 day old twin delivered by caesarean section without prior rupture of the chorioamnion and from the pharynx of her brother. The means by which $C$ trachomatis causes such infection is not known.

Chlamydia trachomatis infects about two thirds of singleton infants born vaginally to infected women. ${ }^{12}$ Normal vaginal delivery may permit prolonged exposure of the infant's head to the infected cervix or endometrium. This exposure permits chlamydiae to enter the conjunctiva, nasopharynx, oropharynx, and lung - anatomical sites commonly found to be infected in exposed infants. Chlamydial infection in infants delivered by caesarean section with unruptured membranes has a less obvious mechanism of transmission, as shown by the following case.

\section{Case report}

Dizygotic twins were born to a black woman aged 29, who had previously delivered a set of twins by caesarean section and was experiencing her sixth pregnancy. Uterine contractions began 10 days before delivery, but labour was arrested with ritodrine. The mother was also treated with betamethasone and magnesium sulphate and with $2 \mathrm{~g}$ cephazolin intravenously 18, 12, and 6 hours before delivery. Labour resumed three hours after the last dose of cephazolin, and 186 minutes before the performance of an elective classical caesarean section. The chorioamnionic membranes were apparently intact until they were incised one minute before delivery of the first twin, who presented cephalically. The presenting parts of the second twin were the feet; he was born one minute after his sister. The placenta was removed one minute later. Both infants were premature, but neither required resuscitation (table 1). In the intensive care nursery, both twins received $1 \%$ silver nitrate solution as eye prophylaxis. The first twin received prophylaxis

\footnotetext{
Address for reprints: Dr Thomas A Bell, Department of Epidemiology, SC-36 University of Washington, Seattle, Washington 98195, USA
}

Accepted for publication 3 April 1988
Table 1 Perinatal histories of dizygotic twins delivered by caesarean section

\begin{tabular}{lrr}
\hline & Twin A & Twin B \\
\hline Gender & Girl & Boy \\
Duration of labour (minutes) & 187 & 188 \\
Time from incision of membrane (minutes) & 1 & 2 \\
Presenting part & Head & Feet \\
Birth mass (grams) & 1740 & 1950 \\
Apgar score at 1 minute & 8 & 7 \\
Apgar score at 5 minutes & 9 & 9 \\
\hline
\end{tabular}

at 33 minutes; no time was recorded for the second. A bilateral tubal ligation was performed on the mother, and she left the hospital four days after delivery. Histological examination of the placenta showed that it was dichorionic and diamnionic. Chorioamnionitis was noted in one section of the membranes. The resected segments of the fallopian tubes were normal.

At six days, the infant girl was noted to have a purulent discharge from the left eye. Culture of the material from the left lower palpebral conjunctiva taken the next day yielded a heavy growth of $C$ trachomatis. ${ }^{3}$ At 10 days, calcium alginate urethrogenital applicators (Hardwood Products, Guilford, Maine, USA) were used to obtain samples from both lower palpebral conjunctivae, the posterior oropharynx, both sides of the posterior nasopharynx, the rectum, and the vagina of the first twin and from her symptomless brother. Cultures from both eyes, both sides of the nasopharynx, and the anus of the girl yielded chlamydiae. The only positive cultures from her brother were those from the nasopharynx and oropharynx. The specimens from the girl generally grew more inclusions than those from the boy (table 2). Treatment of the first twin with oral erythromycin was begun before her discharge from hospital, but the mother and second twin were treated by referring doctors after their discharge from hospital. No cul- 
Table 2 Numbers of inclusion forming units of Chlamydia trachomatis isolated from various anatomical sites of each of a pair of dizygotic twins

\begin{tabular}{lcc}
\hline Anatomical site & Twin $A$ & Twin B \\
\hline Right eye & 4500 & 0 \\
Left eye & 2250 & 0 \\
Right nasopharynx & 36 & 1 \\
Left nasopharynx & 36 & 72 \\
Oropharynx & 0 & 2 \\
Rectum & 1 & 0 \\
Vagina & 0 & (Boy) \\
\hline
\end{tabular}

tures were taken from the mother, who had already been discharged and lived elsewhere.

\section{Discussion}

This report shows that $C$ trachomatis may be acquired through intact membranes by infants delivered by caesarean section. Most rectal infections in infants do not appear before the second month of life. ${ }^{14}$ The early detection of $C$ trachomatis in the rectum of the first twin suggests that her infection was acquired before or at birth. It is not clear whether chlamydiae passed through her gastrointestinal tract or were inoculated direct into the rectum during delivery. In other reports, the respiratory tract, which includes the conjunctiva, is the anatomical site most commonly infected. $^{124}$

Four other infants have been reported as having acquired $C$ trachomatis after caesarean delivery with intact membranes, as shown by isolating the organism from the infants. ${ }^{5-8}$ In another three cases, the method of detection was either isolation or staining with fluorescein labelled monoclonal antibodies. ${ }^{9}$ The small number of such reports suggests that infection after caesarean delivery is rare because $C$ trachomatis is a relatively common infection in pregnant women and is readily transmitted by vaginal delivery. Some prospective studies of infants born to infected women excluded those delivered by caesarean section. ${ }^{12}$ I know of no prospective studies that have assessed the risk of infection by various routes of delivery.

It is surprising that $C$ trachomatis can infect infants when the membranes are ruptured only at caesarean section because amniotic fluid inhibits the growth of chlamydiae. ${ }^{10}$ Perhaps infection is by infected amnionic cells floating in the amnionic fluid." Although the oculogenital biovars of $C$ trachomatis do not usually penetrate epithelial surfaces, chlamydiae have been isolated from chorion after removal of the amnion. ${ }^{12}$ That $C$ trachomatis can infect infants in utero is also suggested by the isolation of the organism from an infant at birth. ${ }^{8}$ Specimens of cord blood from 18 infants with chlamydial conjunctivitis contained no specific IgM, ${ }^{13}$ and thus gave no evidence of prenatal infection.

Chlamydial conjunctivitis, which is usually apparent clinically, has been reported in twins. ${ }^{14}$ The report published here shows that the other twin should be tested when one is found to be infected, regardless of the route of delivery and clinical findings.

This work was supported by US Public Health Service grants EY-00219 and AI-12192. Dr Walter E Stamm graciously performed cultures for Chlamydia trachomatis.

\section{References}

1 Schachter J, Grossman M, Sweet RL, Holt J, Jordan C, Bishop E. Prospective study of perinatal transmission of Chlamydia trachomatis. JAMA 1986;255:3374-7.

2 Bell TA, Sandström KI, Gravett MG, et al. Comparison of silver nitrate solution and erythromycin ointment for prevention of natally acquired Chlamydia trachomatis. Sex Transm Dis 1987; 14:195-200.

3 Stamm WE, Tam R, Koester M, Cles L. Detection of Chlamydia trachomatis inclusions in McCoy cell cultures with fluoresceinconjugated monoclonal antibodies. J Clin Microbiol 1983;17:666-8.

4 Bell TA, Stamm WE, Kuo C-C, Wang S-P, Holmes KK, Grayston JT. Delayed appearance of Chlamydia trachomatis infections acquired at birth. Pediatr Infect Dis 1987;6:928-31.

5 Givner LB, Rennels MB, Woodward CL, Huang S-W. Chlamydia trachomatis infection in infant delivered by caesarean section. Pediatrics 1981;68:420-1.

6 Mårdh P-A, Johansson PJH, Svenningsen N. Intrauterine lung infection with Chlamydia trachomatis in a premature infant. Acta Paediatr Scand 1984;73:569-72.

7 LaScolea LJ, Paroski JS, Burzynski L, Faden HS. Chlamydia trachomatis infection in infants delivered by caesarean section. Clin Pediatr (Phila) 1984;23:118-20.

8 Attenburrow AA, Barker CM. Chlamydial pneumonia in the low birthweight neonate. Arch Dis Child 1985;60:1169-72.

9 Barry WC, Teare EL, Uttley AHC, et al . Chlamydia trachomatis as a cause of neonatal conjunctivitis. Arch Dis Child 1986;61:797-9.

10 Wølner-Hanssen P, Weström L, Mårdh P-A. Influence of amniotic fluid on the formation of chlamydial inclusions in McCoy cell cultures. In: Mårdh P-A, Holmes KK, Oriel JD, Piot P, Schachter J, eds: Chlamydial infections. Amsterdam: Elsevier Biomedical Press 1982;283-6.

11 Harrison HR, Riggin RT. Infection of untreated primary human amnion monolayers with Chlamydia trachomatis. J Infect Dis 1979;140:968-71.

12 Schachter J. Isolation of Bedsoniae from human arthritis and abortion tissues. Am J Ophthalmol 1967;63(suppl):1082-6. (Supplement title Trachoma and allied diseases.)

13 Persson K, Rönnerstam R, Svanberg L, Polberger S. Neonatal chlamydial conjunctivitis. Arch Dis Child 1986;61:565-8.

14 Keskey TS, Suarez M, Gleicher N, Friberg J, Levy HB. Chlamydia trachomatis infections in infants. Mt Sinai $J$ Med $(N Y)$ 1986;53:77-9. 